\title{
LONGITUD DEL CANAL CERVICAL UTERINO COMO FACTOR DE RIESGO DE PARTO PREMATURO EN PACIENTES SINTOMÁTICAS
}

\author{
Pablo Torres Ch. ${ }^{1,2}$, Jorge Carrillo T. ${ }^{1,2}$, Juan Rojas B. ${ }^{1,2}$, Priscila Astudillo A. ${ }^{1,2}$, Iris \\ Delgado A. ${ }^{2}$, Juan Pastén R. ${ }^{3}$, Álvaro Insunza F. ${ }^{1,2}$, Enrique Paiva W. 1,2 \\ 1 Unidad de Gestión Clínica de la Mujer y el RN, Hospital Padre Hurtado. ${ }^{2}$ Facultad de Medicina, Clínica Alemana-Uni- \\ versidad del Desarrollo. 3 Servicio de Neurología, Hospital Barros Luco-Trudeau.
}

\section{RESUMEN}

Objetivo: Determinar si el acortamiento del cuello uterino en pacientes sintomáticas es predictor de parto prematuro en o antes de las 35 semanas y dentro de 7 días. Método: Se estudiaron 852 pacientes que consultaron por dinámica uterina, a las que se midió el cuello uterino al ingreso. Las pacientes fueron manejadas de acuerdo a las Guías Clínicas del Hospital. Se excluyeron las pacientes en trabajo de parto, con membranas rotas o con cerclaje. Resultados: La edad gestacional promedio al ingreso fue 31,5 semanas (rango: 24,0-34,9). Se presentó parto prematuro a las 35 semanas o antes en 61 casos $(7,2 \%)$, y dentro de los 7 días del ingreso en 14 pacientes (1,6\%). Fueron predictores independientes significativos (OR; IC95\%) para un parto $\leq 35$ semanas las siguientes variables: antecedente de prematurez $(2,03 ; 1,06-3,89)$, metrorragia $(6,87 ; 2,83-16,65)$ y canal cervical $<25 \mathrm{~mm}(3,31 ; 1,92-5,7)$. El análisis con curvas ROC demostró que el valor de corte que mejor predice un parto $\leq 35$ semanas y dentro de 7 días de ingresada, es un cuello uterino $<19,5 \mathrm{~mm}(\mathrm{p}<0,05)$. Conclusiones: El acortamiento del canal cervical en pacientes sintomáticas, particularmente con un canal $<20 \mathrm{~mm}$, se asocia con un riesgo significativo de parto prematuro $\leq 35$ semanas y dentro de 7 días de ingresada. La medición de canal cervical ayuda a distinguir a las pacientes en riesgo, con el fin de concentrar esfuerzos en ese grupo de embarazadas.

\section{PALABRAS CLAVE: Parto prematuro, ultrasonido, cuello uterino, factores de riesgo}

\section{SUMMARY}

Objective: To determine if the shortening of cervical length $(C L)$ in women with threatened preterm labor could predict delivery at or before 35 weeks' gestation and within 7 days of presentation. Method: Sonographic measurement of $C L$ was done in 852 women with singleton pregnancies presenting with painful uterine contractions. Women in labor, with ruptured membranes or those with cervical cerclage were excluded. Results: Median gestational age at presentation was 31.5 (range: $24.0-34.9$ ) weeks and median cervical length was 31.5 (range: $3-61$ ) mm. Delivery within 7 days occurred in $14(1.6 \%)$ and delivery at or before 35 weeks, in $61(7.2 \%)$ cases. Significant $(\mathrm{OR} ; 95 \% \mathrm{Cl})$ independent predictors of delivery at or before 35 weeks were vaginal bleeding $(6.87 ; 2.83-16.65)$, cervical length $(3.31 ; \mathrm{Cl} 1.92-5.70)$ and a history of preterm birth $(2.03$; 1.06-3.89). ROC curve analysis showed that a cervical length shorter than $19.5 \mathrm{~mm}(p<0.05)$ was the best cutoff value for predicting a delivery at or before 35 weeks' gestation and within 7 days of presentation. Conclusions: Shortening of cervical length in threatened preterm labor, especially when shorter than $19.5 \mathrm{~mm}$, is associated with a significant risk of preterm labor $\leq 35$ weeks' gestation and within 7 days of admission. 
Sonographic measurement of cervical length helps to recognize patients at risk for preterm birth, in order to focus interventions in that group of patients.

\section{KEY WORDS: Preterm labor, ultrasound, uterine cervix, risk factors}

\section{INTRODUCCIÓN}

La incidencia de parto prematuro (PPt) fluctúa entre 5 y $11 \%(1,2)$ y ha permanecido estable o en ascenso durante los últimos años, a pesar de diversas estrategias dirigidas a su prevención o reducción. Excluyendo las malformaciones congénitas, el parto prematuro es responsable del $60-80 \%$ de la morbimortalidad perinatal $(1,2)$. Esta a su vez, está en directa relación con el peso y edad gestacional del recién nacido $(3,4)$.

El PPt es una condición que se origina a partir de procesos patológicos de naturaleza multifactorial, que activan extemporáneamente uno o más de los componentes que convergen en la vía final común del parto $(5,6)$.

Los componentes uterinos de la vía final común abarcan la contractilidad uterina, la maduración cervical (dilatación y borramiento) y la activación de las membranas deciduales. En esa línea de trabajo, la evaluación ultrasonográfica transvaginal (USTV) del canal cervical y la detección de fibronectina oncofetal han cobrado relevancia al momento de reconocer pacientes en riesgo de PPt (6).

Una estrategia promovida por las autoridades sanitarias y contempladas en las Garantías Explícitas de Salud (GES) sobre prematurez, ha sido concentrar esfuerzos en pacientes que consultan por contracciones uterinas dolorosas (7-17). Poca información se ha publicado en nuestro país sobre la relación entre un canal cervical acortado y el riesgo de PPt en pacientes sintomáticas, particularmente en el lapso de siete días desde la consulta por contracciones. Este estudio tiene como propósito evaluar la asociación entre longitud cervical y parto prematuro en pacientes que consultan por dinámica uterina antes de las 35 semanas de gestación.

\section{MATERIAL Y MÉTODO}

Diseñamos un estudio retrospectivo de todas las pacientes que fueron hospitalizadas con diagnóstico de síntoma de parto prematuro, entre el 1 de junio de 2002 y el 30 de junio de 2005, en la Unidad de Gestión Clínica de la Mujer y el Recién Nacido del Hospital Padre Hurtado (HPH), Área Sur Oriente de Santiago.

Se incluyeron todas las pacientes embarazadas que al momento de la consulta tuvieran una edad gestacional entre 24,0 y 34,9 semanas y se presentaran con dinámica uterina dolorosa, con una frecuencia de 2 o más contracciones en 10 minutos. Se excluyeron las pacientes que al ingreso tuvieran embarazo múltiple, edad gestacional dudosa, rotura prematura de membranas, trabajo de parto, aquellas con cerclaje, desprendimiento prematuro de placenta normoinserta, sufrimiento fetal agudo, corioamnionitis, diagnóstico prenatal de malformación incompatible con la vida, u otra patología obstétrica o médica que requiriera interrupción inmediata del embarazo.

Las pacientes eran evaluadas al consultar cuando tenían contracciones uterinas, se les medía el canal cervical con transductor transvaginal según técnica estandarizada (18): paciente en posición ginecológica, vejiga vacía, tres mediciones desde el orificio cervical interno al externo, se seleccionaba la medición más corta. Una vez hospitalizadas, el protocolo de manejo fue el siguiente:

1.Hidratación con solución Ringer Lactato o solución fisiológica durante 3 horas (500cc la primera hora, $300 \mathrm{cc}$ en la segunda y tercera hora).

2.Si al cabo de ese lapso no había cedido la dinámica uterina, se comenzaba una infusión de sulfato de magnesio a $2 \mathrm{~g} /$ hora y se administraba betametasona $12 \mathrm{mg} \mathrm{IM}$ cada 24 horas por 2 veces.

3.Si luego de tres horas de infusión de sulfato de magnesio las contracciones uterinas no cedían, se cambiaba a fenoterol, previa amniocentesis para descartar infección intraamniótica.

a.En caso de comprobarse una infección intramniótica, se suspendía la tocólisis y se iniciaban antibióticos.

b.En caso de descartarse la infección intramniótica la frenación se mantenía hasta 12 horas después de un control sin dinámica uterina.

4.Este protocolo se utilizó hasta el año 2003. A partir de ese año se dejó de usar el sulfato de magnesio y sólo se indicó fenoterol como tocolítico, el cual se iniciaba luego de la hidratación fallida. Desde esa fecha el sulfato de magnesio sólo fue indicado en aquellas pacientes con contraindicación de uso de betamiméticos o en algunas pacientes en que había fracasado el uso de fenoterol.

Las variables analizadas se extrajeron de la base de datos computacional de los egresos de 
la Sección de Embarazo Patológico: edad materna, fecha de hospitalización, fecha de egreso, días de hospitalización, edad gestacional al ingreso y al egreso, longitud del cuello al ingreso, uso de tocolíticos, uso de corticoides, necesidad de amniocentesis. Adicionalmente se revisaron todas las fichas clínicas, donde se ratificó o rectificó la información obtenida de la base de datos y se extrajo información referente a la previsión, escolaridad, estado civil, antecedentes mórbidos, de tabaquismo o drogadicción, forma de cálculo de la edad gestacional y antecedentes obstétricos. También se obtuvo información del motivo de consulta, frecuencia de dinámica uterina al ingreso, longitud cervical, manejo de la dinámica uterina (hidratación, sulfato de magnesio, fenoterol), resultado de amniocentesis, fecha de corticoides, fecha de parto, edad gestacional al parto, vía del parto, causa si fue parto quirúrgico, peso del RN, Apgar al minuto 1 y 5, y complicaciones neonatales.

Definimos como objetivo principal del estudio el determinar si la longitud cervical medida por ultrasonido predice parto de pretérmino en o antes de las 35 semanas. Como objetivo secundario quisimos determinar si predice parto prematuro dentro de siete días desde el ingreso.

Se utilizó test de Chi cuadrado o Fisher para variables categóricas y test $T$ de Student para variables continuas. En el caso de las variables que demostraron significancia estadística, se hizo análisis de regresión logística bivariable, para ratificar el resultado. Posteriormente, se hizo un análisis de regresión logística multivariable con las variables que fueron significativas en el análisis bivariable, para correlacionarlas con la ocurrencia de un parto $\leq 35$ semanas y dentro de 7 días. Para determinar el mejor valor de corte entre la longitud de canal cervical y la ocurrencia de un parto en o antes de las 35 semanas y dentro de 7 días, se ejecutaron curvas de ROC. Para todos los análisis se consideró como valor de significancia estadística un $\mathrm{p}<0,05$.

\section{RESULTADOS}

En el Hospital Padre Hurtado, en el período de estudio hubo un total de 19.937 partos. La tasa de parto por ocurrencia, en o antes de 35 semanas, fue de $3,2 \%$ (639/19.937). Reunieron los criterios de ingreso al estudio 883 pacientes que consultaron por contracciones uterinas; en 31 pacientes no fue posible obtener la información del parto, por no ocurrir en la institución (3,5\% de pérdida de la cohorte), por lo que se analizaron 852 pacientes.

En la Tabla I se describen las características sociodemográficas y al ingreso de las pacientes. La edad gestacional promedio al ingreso fue 31,5 semanas (rango: 24.0-34.9); 61 embarazadas $(7,2 \%)$ presentaron parto prematuro en o antes de las 35 semanas, mientras que 14 pacientes $(1,6 \%)$ presentaron su parto dentro de los 7 días del ingreso.

Las variables obstétricas de la población se describen en la Tabla II. Destaca el incremento de la frecuencia de partos prematuros en la medida que se acorta el canal cervical: $15,4 \%, 24,2 \%$ y 45,4 $\%$, cuando el canal es menor de 25, 20 y $15 \mathrm{~mm}$, respectivamente. Treinta y seis de las 61 pacientes $(59 \%)$ con PPt $\leq 35$ semanas tenían un cérvix de $25 \mathrm{~mm}$ o mayor. Un total de 380 pacientes $(44,6 \%)$ requirieron frenación, de éstas, el $97 \%$ recibió corticoides durante la hospitalización y a 81 pacientes $(21,3 \%)$ se les practicó amniocentesis, resultando positiva para infección intraamniótica en 1 caso. La vía del parto fue: espontáneo $68,1 \%$; fórceps $9,1 \%$; cesárea $22,8 \%$.

En el análisis de regresión logística bivariable, al correlacionar la ocurrencia de parto prematuro $\leq 35$ semanas con las diferentes variables estudiadas, demostraron significancia estadística el antecedente de parto prematuro, la metrorragia y la longitud cervical tanto $<25,<20$ y $<15 \mathrm{~mm}$ (Tabla III). Al repetir el análisis para la ocurrencia del parto dentro de 7 días del ingreso, se encontró una asociación con metrorragia y cuello corto, tanto $<25$, $<20$ y $<15 \mathrm{~mm}$. No hubo correlación significativa con edad, escolaridad, estado civil, previsión, índice de masa corporal, tabaquismo o la paridad de las pacientes, tanto para un parto prematuro dentro de 7 días del ingreso, como para su ocurrencia en o antes de las 35 semanas.

En el análisis de regresión logística multivariable (OR; IC 95\%), fueron factores independientes para riesgo de parto prematuro $\leq 35$ semanas, la metrorragia $(7,27 ; 2,8-18,9)$, un canal cervical $<15$ $\mathrm{mm}(6,0 ; 1,72-21,1)$ y el antecedente de parto prematuro $(2,4 ; 1,2-4,8)$ (Tabla IV). Para la ocurrencia de un parto dentro de 7 días del ingreso, ninguna de las variables se demostró como factor independiente (Tabla IV). Los índices diagnósticos demostraron que la longitud de canal cervical tiene una alta especificidad y valor predictivo negativo, y una baja sensibilidad y valor predictivo positivo, tanto para la predicción de parto prematuro $\leq 35$ semanas, como para la detección de un parto dentro de 7 días desde el ingreso (Tabla $\mathrm{V}$ ).

El análisis con curvas ROC determinó que un cuello de $19,5 \mathrm{~mm}$ era el mejor valor de corte para la predicción de parto prematuro, tanto para $\leq 35$ semanas como para su ocurrencia dentro de 7 días (Figuras 1 y 2 ). 
Tabla I

CARACTERÍSTICAS SOCIO-DEMOGRÁFICAS Y AL INGRESO DE LAS PACIENTES (N: 852)

Edad, años (media \pm DS)

Escolaridad (n: $\left.842^{*}\right)$

Ninguna o básica

Media (completa o incompleta)

Superior

Estado civil (n: $\left.847^{\star}\right)$

Casada o unión estable

Soltera o separada

FONASA A-B

FONASA C-D o ISAPRE

IMC (Media \pm DS)

Tabaquismo activo en el embarazo (n: 638*)

Paridad

Nulíparas

Multíparas

Historia de parto prematuro previo

EG al ingreso, semanas (media \pm DS)

Motivo de consulta

$\mathrm{DU} \geq 2 / 10 \mathrm{~min}$

Metrorragia (c/DU)

Otro motivo de consulta

Longitud $(\mathrm{mm})$ cervical al ingreso, (media $\pm \mathrm{DS}$ )
$24,1 \pm 6,52$

$250(29,7 \%)$

$562(66,7 \%)$

$30(3,6 \%)$

$560 *(66,1 \%)$

$287(33,9 \%)$

$696(81,8 \%)$

$154(18,2 \%)$

$28,3 \pm 4,63$

$124(19,4 \%)$

$377(44,2 \%)$

$475(55,8 \%)$

$106 / 475(22,3 \%)$

$31,1 \pm 2,66$

$775(91,0 \%)$

$25(2,9 \%)$

$52(6,1 \%)$

$31,5 \pm 8,02$

$162(19,0 \%)$

$66(7,7 \%)$

$22(2,6 \%)$

*: Indica data incompleta. Todos los valores expresados en número (\%), excepto cuando sea indicado en la tabla. DU: dinámica uterina. IMC: índice de masa corporal

\section{Tabla II}

RESULTADOS OBSTÉTRICOS DE LA POBLACIÓN EN ESTUDIO ( $\mathrm{N}=852$ )

EG al parto, semanas (media \pm DS)

Momento del parto prematuro

Parto prematuro según longitud de cervix

$$
\begin{aligned}
& \text { cuello }<25 \\
& \text { cuello }<20 \\
& \text { cuello }<15
\end{aligned}
$$

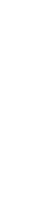


Tabla III

RESULTADOS DEL ANÁLISIS BIVARIABLE PARA LA PREDICCIÓN DE PARTO PREMATURO EN O ANTES DE 35 SEMANAS Y DENTRO DE 7 DÍAS

\begin{tabular}{lcc}
\hline Variable & Parto 35 semanas & Parto dentro de 7 días \\
\hline Antecedentes de prematurez & $2,03(1,06-3,89)$ & $2,86(0,88-9,37)^{\star}$ \\
Metrorragia & $6,87(2,83-16,65)$ & $5,90(1,24-27,91)$ \\
Cuello $<25 \mathrm{~mm}$ & $3,31(1,92-5,70)$ & $5,92(2,02-17,31)$ \\
Cuello $<20 \mathrm{~mm}$ & $5,26(2,78-9,97)$ & $7,07(2,30-21,77)$ \\
Cuello $<15 \mathrm{~mm}$ & $12,72(5,24-30,86)$ & $11,75(3,03-45,58)$
\end{tabular}

Resultados expresados en OR (95\% IC). *: no significativo.

\section{Tabla IV}

RESULTADO DEL ANÁLISIS DE REGRESIÓN LOGÍSTICA MULTIVARIABLE PARA LA PRESENTACIÓN DE UN PARTO EN O ANTES DE 35 SEMANAS Y DENTRO DE 7 DÍAS

\begin{tabular}{lcc}
\hline Variable & Parto 35 semanas & Parto dentro de 7 días \\
\hline Antecedentes de prematurez & $2,40(1,21-4,77)$ & - \\
Metrorragia & $7,27(2,78-18,97)$ & $4,61(0,86-24,58)^{\star}$ \\
Cuello $<25 \mathrm{~mm}$ & $1,94(0,89-4,22)^{\star}$ & $3,90(0,95-15,97)^{\star}$ \\
Cuello $<20 \mathrm{~mm}$ & $1,32(0,42-4,13)^{\star}$ & $1,25(0,19-8,04)^{\star}$ \\
Cuello $<15 \mathrm{~mm}$ & $6,02(1,72-21,10)$ & $3,26(0,48-21,78)^{\star}$
\end{tabular}

Resultados expresados en OR (95\% IC). *: no significativo.

Tabla V

ÍNDICES DIAGNÓSTICOS EN LA PREDICCIÓN DE PARTO EN O ANTES DE 35 SEMANAS Y DENTRO DE 7 DÍAS

Sensibilidad

(\%)

35 semanas
Especificidad

(\%)

\section{2}

94

99 


\section{Curva COR}

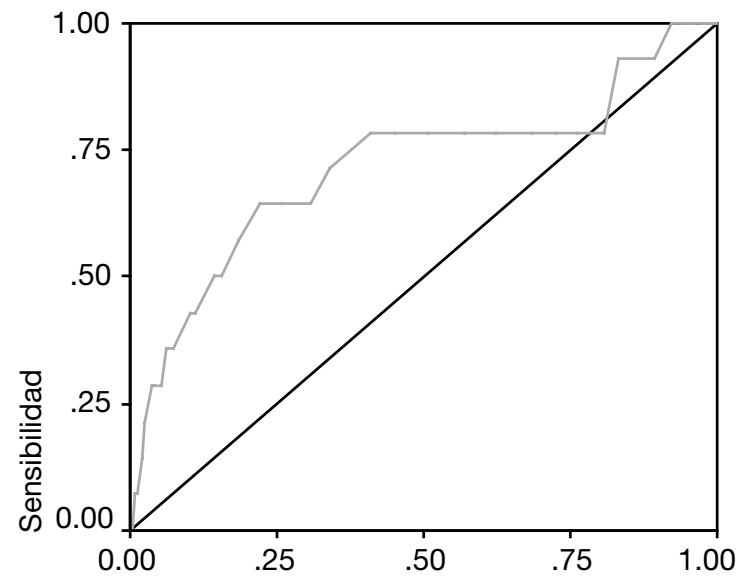

1 - Especificidad

Los segmentos diagonales son producidos por los empates.

Figura 1: Curva ROC para la predicción de parto prematuro antes de 7 días. El mejor valor de corte se ubica en 19,5 mm de longitud cervical. (Área: $0,71 ; p=0,005)$.

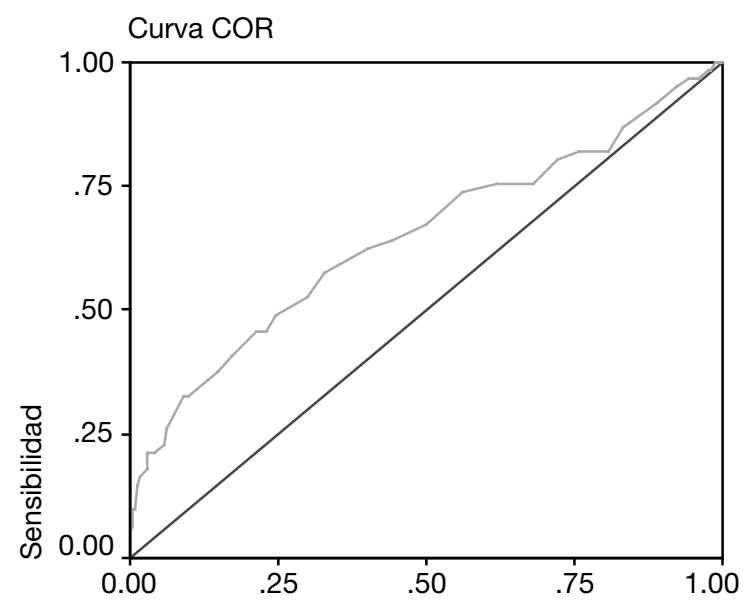

1 - Especificidad

Los segmentos diagonales son producidos por los empates.

Figura 2: Curva ROC para la predicción de parto prematuro a las 35 semanas o anterior. El mejor valor de corte se ubica en 19,5 mm de longitud cervical. (Área: 0,646; $p=0,01$ ).

\section{DISCUSIÓN}

Las recientes políticas del Ministerio de Salud relativas a la prevención de parto prematuro en el Plan de Garantías Explícitas en Salud (GES), re- comiendan observación, durante 2 horas, de las pacientes con dinámica uterina igual o mayor a 3 contracciones en 30 minutos. Si la actividad uterina cede, se debe dar de alta a la paciente con indicaciones de seguir su control en el nivel asistencial correspondiente. Si la dinámica uterina persiste, en ausencia de modificaciones cervicales, se mide el canal cervical con un transductor ecográfico transvaginal. Luego, la embarazada se somete a un protocolo de manejo según la guía clínica (19). Nuestro estudio analizó los resultados de la hospitalización de un grupo de pacientes ingresadas con dinámica uterina de $2 / 10$ minutos o más, en un período de 37 meses.

Los resultados demuestran que la medición de la longitud del cuello uterino es un instrumento eficaz para detectar riesgo significativo de parto prematuro con edad gestacional $\leq 35$ semanas, y dentro de 7 días desde el ingreso. Un canal cervical menor de $25 \mathrm{~mm}$, menor de $20 \mathrm{~mm}$ y menor de 15 $\mathrm{mm}$, se asoció significativamente con riesgo de parto prematuro: a mayor acortamiento del canal cervical, mayor incidencia de parto prematuro, lo que ya demostró lams y cols hace más de diez años (17), evaluando canal cervical en una población de pacientes asintomáticas evaluadas entre las $21 \mathrm{y}$ 25 semanas de gestación. En nuestra población se reafirma además que un canal cervical menor de 15 $\mathrm{mm}$ tiene un riesgo superior a $45 \%$ de terminar en parto prematuro en o antes de las 35 semanas, lo que coincide con el estudio de Tsoi y cols efectuado en 2005 (9).

Tal como lo reporta la literatura $(9,10)$, la metrorragia es un signo clínico que debe ser considerado de importancia, pues se encontró un riesgo 7 veces superior de terminar en un parto en o antes de las 35 semanas. Otro factor de riesgo significativo para parto prematuro fue el antecedente de uno previo (15).

Es llamativo que el análisis con curvas ROC haya determinado que un canal cervical de 19,5 $\mathrm{mm}$ sea el valor de corte que mejor predice parto prematuro para los dos resultados en estudio; esta cifra es similar a la longitud de cuello $(20 \mathrm{~mm})$ que se ha usado empíricamente en el Hospital Padre Hurtado para mantener hospitalizadas a las pacientes hasta las 35 semanas una vez cesadas las contracciones uterinas.

Comparando nuestros resultados con los de otros autores, podemos señalar que Tsoí y cols (9) obtuvieron una tasa de $14,9 \%$ y $8,4 \%$ de parto antes de las 35 semanas y dentro de 7 días, y demostraron que un canal cervical menor o igual a $15 \mathrm{~mm}$ se asociaba con un $44,7 \%$ y un $29,8 \%$ de parto prematuro antes de las 35 semanas y a 7 días, respectivamente. Gómez y cols (8) obtuvieron 
una tasa de $20,0 \%$ y $13,0 \%$ de parto antes de las 35 semanas y dentro de 7 días, y comunicó que un canal cervical $\leq$ a $15 \mathrm{~mm}$ se asociaba con un $63,3 \%$ y $56,7 \%$ de parto prematuro antes de las 35 semanas y a 7 días, respectivamente.

Consideramos que una fortaleza de este trabajo es el volumen de la cohorte, que de acuerdo a nuestra revisión bibliográfica es la de mayor casuística publicada. Otra fortaleza de este estudio ha sido contar con un manejo homogéneo, estandarizado y en equipo de las pacientes, basado en Guías Clínicas, cuya adherencia es periódicamente auditada en las visitas clínicas de la Institución. La pérdida de información correspondió a solo 31 pacientes $(3,5 \%)$.

Como potenciales limitaciones del estudio debemos mencionar el carácter retrospectivo del análisis. Sin embargo, el registro de nuestra base de datos es rigurosa y auditada periódicamente, lo que hace improbable algún sesgo de selección de pacientes o de información incompleta.

En el período de estudio, en el Hospital Padre Hurtado se verificaron 19.937 partos; de ellos, 639, correspondientes al 3,2\%, tenían $\leq 35$ semanas. La tasa de parto prematuro en nuestra cohorte de pacientes sintomáticas, fue de $7,2 \%$, más del doble que la población general; esto respalda la necesidad de cuidados dirigidos en este grupo de pacientes, especialmente cuando la longitud cervical es menor a $20 \mathrm{~mm}$. Sin embargo, aún con esta precaución, estamos lejos de resolver el problema clínico del parto prematuro: las 61 pacientes de nuestra cohorte, que finalmente evolucionaron hacia un parto prematuro, representan solamente $9,5 \%$ del total de pacientes con parto prematuro en ese período (639 casos). Un gran porcentaje no pudo ser identificado para tomar acciones adecuadas como el uso oportuno de corticoides y/o una atención en un centro de cuidado neonatal especializado.

\section{CONCLUSIÓN}

El acortamiento del canal cervical en pacientes sintomáticas, particularmente con un canal $<20$ $\mathrm{mm}$, se asocia con un riesgo significativo de parto prematuro $\leq 35$ semanas y dentro de 7 días de ingresada. La medición de canal cervical ayuda a distinguir a las pacientes en riesgo, con el fin de concentrar esfuerzos en ese grupo de embarazadas.

\section{BIBLIOGRAFÍA}

1. Goldenberg R. Management of preterm labor. Obstet Gynecol 2002;100(5):1020-37.

2. lams J. Prediction and early detection of preterm labor. Obstet Gynecol 2003;101(2):402-12.

3. Morgues M, Henríquez M, Tohá D, Vernal P, Pittaluga
E, Vega S, et al. Sobrevida del niño menor de $1500 \mathrm{~g}$ en Chile. Rev Chil Obstet Ginecol 2002;67(2):100-5.

4. Hübner M, Ramírez R. Sobrevida, viabilidad y pronóstico del prematuro. Rev Med Chile 2002;130(8):9318.

5. Lockwood C. Predicting premature delivery - No easy task. N Engl J Med 2002;346(4):282-4.

6. Lockwood C, Kuczynski E. Markers of preterm delivery risk. Ultrasound Obstet Gynecol 1998;12:301-3.

7. Jankelevich J, Grafia M. Evaluación ultrasonográfica del cérvix uterino en la detección de pacientes de alto riesgo para parto prematuro. Rev Chil Ultrasonog 1998;1(1):13-8.

8. Gómez R, Romero R, Medina L, Nien J, Chaiworapongsa $\mathrm{T}$, Carstens $\mathrm{M}$, et al. Cervicovaginal fibronectin improves the prediction of preterm delivery based on sonographic cervical length in patients with preterm uterine contractions and intact membranes. Am J Obstet Gynecol 2005;192(2):350-9.

9. Tsoi E, Fuchs I, Rane S, Geerts L, Nicolaides K. Sonographic measurement of cervical length in threatened preterm labor in singleton pregnancies with intact membranes. Ultrasound Obstet Gynecol 2005;25(4): 353-6.

10. Fuchs I, Henrich W, Osthues K, Dudenhausen J. Sonographic cervical length in singleton pregnancies with intact membranes presenting with threatened preterm labor. Ultrasound Obstet Gynecol 2004;24(5):554-7.

11. Tsoi E, Akmal S, Rane S, Otigbah C, Nicolaides K. Ultrasound assessment of cervical length in threatened preterm labor. Ultrasound Obstet Gynecol 2003;21(6):5525.

12. Honest H, Bachmann L, Coomarasamy A, Gupta J, Kleijnen J, Khan K. Accuracy of cervical transvaginal sonography in predicting preterm birth: a systematic review. Ultrasound Obstet Gynecol 2003;22:305-22.

13. Welsh A, Nicolaides K. Cervical screening for preterm delivery. Curr Opin Obstet Gynecol 2002;14(2):195202.

14. To M, Skentou C, Liao A, Cacho A, Nicolaides K. Cervical length and funneling at 23 weeks of gestation in the prediction of spontaneous early preterm delivery. Ultrasound Obstet Gynecol 2001;18:200-3.

15. Hassan S, Romero R, Berry S, Dang K, Blackwell S, Treadwell $\mathrm{M}$, et al. Patients with an ultrasonographic cervical $\leq 15 \mathrm{~mm}$ have nearly a $50 \%$ risk of early spontaneous preterm delivery. Am J Obstet Gynecol 2000;188(6):1458-67.

16. Heath V, Southall V, Souka A, Elisseou A, Nicolaides K. Cervical length at 23 weeks of gestation: prediction of spontaneus preterm dilivery. Ultrasound Obstet Gynecol 1998; 12: 312-7.

17. lams J, Goldenberg R, Meis P, Mercer B, Moawad $A$, Das $A$, et al. The length of the cervix and risk of spontaneous premature delivery. $\mathrm{N}$ Engl $\mathrm{J}$ Med 1996;334(9):567-72.

18. Kagan K, To M, Tsoi E, Nicolaides K. Preterm birth: the value of sonographic measurement of cervical lenght. BJOG 2006;113(Suppl. 3):52-6.

19.Oyarzún E, Parra M, Gómez R, Gonzalez R, Castro R, Ayala A, et al. Guía clínica prevención del parto prematuro, Garantías Explícitas en Salud. Ministerio de Salud Chile 2005. 